\title{
OPEN PEG-modified gadolinium nanoparticles as contrast agents for in vivo micro-CT
}

\author{
Charmainne Cruje ${ }^{1,2}$, P. Joy Dunmore-Buyze ${ }^{1}$, Eric Grolman ${ }^{1,3}$, David W. Holdsworth ${ }^{1,2,3}$, \\ Elizabeth R. Gillies ${ }^{3,4,5,6}$ \& Maria Drangova ${ }^{1,2,3,6 \bowtie}$
}

Vascular research is largely performed in rodents with the goal of developing treatments for human disease. Micro-computed tomography (micro-CT) provides non-destructive three-dimensional imaging that can be used to study the vasculature of rodents. However, to distinguish vasculature from other soft tissues, long-circulating contrast agents are required. In this study, we demonstrated that poly(ethylene glycol) (PEG)-coated gadolinium nanoparticles can be used as a vascular contrast agent in micro-CT. The coated particles could be lyophilized and then redispersed in an aqueous solution to achieve $100 \mathrm{mg} / \mathrm{mL}$ of gadolinium. After an intravenous injection of the contrast agent into mice, micro-CT scans showed blood pool contrast enhancements of at least $200 \mathrm{HU}$ for 30 min. Imaging and quantitative analysis of gadolinium in tissues showed the presence of contrast agent in clearance organs including the liver and spleen and very low amounts in other organs. In vitro cell culture experiments, subcutaneous injections, and analysis of mouse body weight suggested that the agents exhibited low toxicity. Histological analysis of tissues 5 days after injection of the contrast agent showed cytotoxicity in the spleen, but no abnormalities were observed in the liver, lungs, kidneys, and bladder.

Micro-computed tomography (micro-CT) provides a quantitative, non-destructive, fast and cost-effective means of studying vascular disease in mouse models ${ }^{1-9}$. In live mice, micro-CT can provide imaging resolution down to tens of micrometers within tens of minutes. Because CT contrast is derived from the density-dependent attenuation of $\mathrm{x}$ rays, soft tissues (which have similar densities) provide little differential contrast. Hence, $\mathrm{x}$-ray attenuating contrast agents are injected intravenously to distinguish the vasculature from surrounding soft tissues during a micro-CT scan, enabling visualization and quantitative tracking of blood vessels, including during studies of novel therapies for re-vascularization ${ }^{10-12}$. For optimal utility in in vivo imaging, contrast agents must have a high initial concentration of a highly attenuating contrast element, circulate in the blood pool during the course of the micro-CT scan, and be cleared from the body after imaging to enable longitudinal studies with repeated injections. Clinically used contrast agents comprise small molecules that are cleared renally within seconds of intravenous administration; while these contrast agents meet the requirements of human imaging where scan times are short, in vivo micro-CT protocols require circulation times in the order of tens of minutes ${ }^{13-17}$.

Advances in nanotechnology and polymer science have enabled the development of commercially available agents that can evade immediate clearance from the blood-pool and circulate for prolonged periods in mice ${ }^{16,18}$. These agents are composed of nanoparticles with diameters greater than $10 \mathrm{~nm}$, thus avoiding clearance via the kidneys $s^{5,19-22}$. To further evade clearance by the reticuloendothelial system (RES), carrier polymers that act as shields from the in vivo milieu are used to coat the nanoparticles. This "core-shell" design can also be used to deliver a high loading of contrast material in the core, making the design important for micro-CT contrast agents, where contrast-agent concentrations of at least $100 \mathrm{mg} / \mathrm{mL}$ are typically required. Poly(ethylene glycol) (PEG) has been widely utilized to coat nanoparticles to achieve long circulation times ${ }^{23,24}$ because of its stealthy properties with respect to the RES, high water solubility, low cytotoxicity, availability in different lengths, and a terminal group can be modified into functional groups to coat nanoparticles ${ }^{25,26}$. For example, phospholipidterminated PEG is used in Fenestra VC, which encapsulates $50 \mathrm{mg} / \mathrm{mL}$ of iodine within lipid emulsions $\mathrm{s}^{8,20,27,28}$,

\footnotetext{
${ }^{1}$ Robarts Research Institute, Western University, London, ON N6A 5B7, Canada. ${ }^{2}$ Department of Medical Biophysics, Western University, London, ON N6A 5B7, Canada. ${ }^{3}$ School of Biomedical Engineering, Western University, London, ON N6A 5B7, Canada. ${ }^{4}$ Department of Chemistry, Western University, London, ON N6A 5B7, Canada. ${ }^{5}$ Department of Chemical and Biochemical Engineering, Western University, London, ON N6A 5B7, Canada. ${ }^{6}$ These authors contributed equally: Elizabeth R. Gillies and Maria Drangova. ${ }^{\square}$ email: mdrangova@ robarts.ca
} 
while $15 \mathrm{~nm}$ gold nanoparticles are coated with thiol-terminated PEG in AuroVist, enabling contrast loading of $200 \mathrm{mg} / \mathrm{mL}^{29-31}$.

As iodine is the most commonly utilized clinical CT contrast agent, many of the micro-CT vascular agents rely on iodine's attenuating properties. Apart from gold, which has higher attenuation than iodine across the entire energy spectrum, metal-based agents such as alkaline earth metals ${ }^{21}$ and transition metals ${ }^{32}$, have been reported or commercialized. Another class of metals that is ideally suited for micro-CT are the lanthanides (e.g. gadolinium, erbium $)^{33}$, which have $\mathrm{k}$ edges $(38-64 \mathrm{keV})^{34}$ that coincide with the peaks of the x-ray energy distributions typically used in high-resolution in vivo micro-CT scanners (typically operating at below $100 \mathrm{kVp}$ ). The ideal position of the k-edges offers potential to increase contrast attenuation for single-energy micro-CT scans $^{33}$ and, more importantly, offers the opportunity to match the available $\mathrm{x}$-ray spectrum and contrast agent in dual-energy micro-CT, which is used to distinguish contrast-enhanced vessels from inherently high-attenuating tissues (e.g. bone, calcifications) ${ }^{35}$.

Lanthanide agents designed for in vivo vascular micro-CT are not commercially available, but studies are emerging demonstrating the utility of PEG-modified lanthanide nanoparticles in microimaging ${ }^{36-39}$. To prepare lanthanides that can be used in the in vivo milieu in small animals, common methods include the use of clinically-available MRI agents to formulate nanoparticles that evade rapid renal clearance, or the synthesis of hydrophobic lanthanide nanoparticles encapsulated within a shell of phospholipid-polymer conjugate molecules (i.e. liposomes or lipid nanoparticles). While suspending high concentrations of lanthanides (i.e. at least $100 \mathrm{mg} /$ $\mathrm{mL}$ ) in an aqueous environments is challenging, we have recently reported the successful synthesis of block copolymer assembly encapsulations of erbium nanoparticles containing $100 \mathrm{mg} / \mathrm{mL}$ of erbium, and demonstrated its utility as a vascular contrast agent when operating at $90 \mathrm{kVp}^{40}$. However, accumulation of the agent within the RES organs resulted in limited viability two days following in vivo contrast agent injection.

The purpose of this work was to synthesize a blood pool contrast agent with at least $100 \mathrm{mg} / \mathrm{mL}$ of lanthanide to be cleared by the RES. It is important to note that the extravascular gadolinium-based contrast agents that are used clinically (mainly in MRI) leave the blood pool within minutes of injection and circulate within the extracellular space, while the nanoparticle-based agent that this study developed was intentionally designed to remain in the vasculature over long periods of time and be cleared before the potential release of free gadolinium ions. Instead of encapsulating multiple nanoparticles in block copolymer assemblies that were greater than $100 \mathrm{~nm}$ in diameter ${ }^{40}$, we hypothesized that an alternative approach of directly modifying the surface of gadolinium nanoparticles (GdNP) with PEG, would result in smaller polymer-coated nanoparticles, thereby assisting GdNP clearance from RES organs. (Note that gadolinium was selected as the lanthanide to evaluate because it is currently the only lanthanide approved for clinical use, in any form). We demonstrate that such agents can be synthesized, lyophilized and then redispersed to achieve $100 \mathrm{mg} / \mathrm{mL}$ of lanthanide in an injectable formulation. Our in vivo results show that the agents provide contrast enhancement values of at least $200 \mathrm{HU}$ in the blood pool for $30 \mathrm{~min}$ before being processed in the liver and spleen. The agents were well tolerated, with some gadolinium retention in clearance organs observed 5 days after a high dose injection. To our knowledge, this is the first report on the successful synthesis of colloidally stable aqueous suspensions of gadolinium-based nanoparticles at $100 \mathrm{mg} / \mathrm{mL}$ that exhibit sufficient circulation times rendering them suitable contrast agents for vascular imaging by micro-CT.

\section{Methods}

Reagents and GdNP synthesis details. Details regarding the reagents and their commercial suppliers are available in the Supporting Information (SI). Further details on synthesis and characterization methods are also presented in the SI.

Contrast agent preparation and physical characterization. Synthesis of GdNP. Oleate (OA)coated $\mathrm{NaGdF}_{4}(\mathrm{OA}-\mathrm{GdNP})$ were synthesized by a previously reported method ${ }^{40}$. Phosphate-terminated $\mathrm{PEG}_{1000}\left(\mathrm{PPEG}_{1000}\right), \mathrm{PEG}_{2000}\left(\mathrm{PPEG}_{2000}\right)$, and $\mathrm{PEG}_{5000}\left(\mathrm{PPEG}_{5000}\right)$, where the subscripts indicate PEG molar mass in $\mathrm{g} / \mathrm{mol}$, were also synthesized as previously reported ${ }^{41}$. Two versions of PPEG-coated GdNP were synthesized-one with $\mathrm{PPEG}_{2000}$ only $\left(\mathrm{PPEG}_{2000}-\mathrm{GdNP}\right)$, and a formulation using both of $\mathrm{PPEG}_{5000}$ and $\mathrm{PPEG}_{1000}$ $\left(P P E G_{5000}-P_{P E G}{ }_{1000}-G d N P\right)$. An overview of the PPEG-coating process is presented in Fig. 1. Specifically, GdNP $(1.0 \mathrm{~g})$ and $\mathrm{PPEG}_{2000}(1.0 \mathrm{~g})$ were each dissolved in $12.5 \mathrm{~mL}$ of tetrahydrofuran (THF), then combined under magnetic stirring. Deionized water $(225 \mathrm{~mL})$ was then added. After stirring for one hour, THF was evaporated and the nanoparticles were purified by dialysis and sterile vacuum filtration (see SI for details). The purified GdNPs were lyophilized and stored at room temperature until they were redispersed immediately prior to use. The same procedure was followed for PPEG $5_{5000}-\mathrm{PPEG}_{1000}$-GdNP, except $\mathrm{PPEG}_{1000}$ (1.0 g) dissolved in THF (12.5 mL) was added and the suspension was stirred for one hour before organic solvent evaporation ${ }^{42}$.

Physical characterization. The hydrodynamic diameters of the GdNPs were measured by dynamic light scattering (DLS) and were visualized by transmission electron microscopy (TEM). The gadolinium concentration in the contrast agent was measured by inductively coupled plasma-mass spectrometry (ICP-MS) and the results were used to determine the mass of dried contrast agent required to achieve gadolinium concentrations of $100 \mathrm{mg} /$ $\mathrm{mL}$. Micro-CT imaging, where image intensity varies linearly with concentration, was also used to verify the contrast agent concentration (details in SI).

Colloidal stability. Saline, which is isotonic with blood, was selected as the solvent for the PPEG-GdNPs. The mouse serum mimic was composed of $\mathrm{pH} 7.4$ phosphate buffered saline, $0.5 \mu \mathrm{g} / \mathrm{mL}$ mouse immunoglobulins, $10 \mathrm{mg} / \mathrm{mL}$ bovine serum albumin and $1 \mathrm{mg} / \mathrm{mL}$ sodium azide. Colloidal stability in a mouse serum mimic 
A

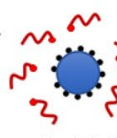

in THF

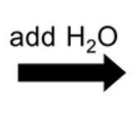

ligand exchange

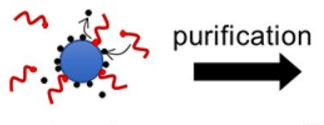

rand exchange

B

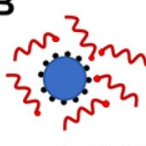

in THF
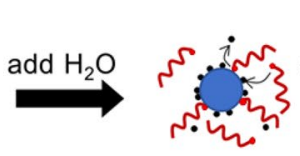

ligand exchange

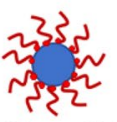

$\mathrm{PPEG}_{2000}-\mathrm{GdNP}$

Figure 1. The synthesis of (A) $P P E G_{2000}-G d N P$ and (B) $P P E G_{5000}-P_{P E G} 1000-G d N P$. OA is displaced by PPEG through ligand exchange, and the solution is purified by dialysis and vacuum sterile filtration to eliminate OA and excess PPEG.

in vitro served as an indicator of stability in the blood in vivo. DLS size measurements were performed on the PPEG-GdNP redispersed at $4 \mathrm{mg} / \mathrm{mL}$ and the average sizes were observed for up to one hour.

Gadolinium release study. To explore the possibility of gadolinium release, $\mathrm{PPEG}_{2000}$-GdNP was dialyzed against $1 \mathrm{mM}$ calcium chloride and $0.01 \mathrm{mM}$ zinc chloride in normal saline as dialysate. The gadolinium concentrations in the dialysate were quantified by ICP-MS $1 \mathrm{~h}, 3$ days, and 5 days after the start of dialysis. Measured concentrations were compared to the gadolinium content of the injected agent.

Micro-CT scanning and image analysis. Scans were performed using a GE Locus Ultra micro-CT scanner (GE Healthcare, London $\mathrm{ON})$. Whole-body mouse images were acquired over 1000 views $\left(360^{\circ}, 16 \mathrm{~ms}\right.$ per view) at $80 \mathrm{kVp}, 55 \mathrm{~mA}$ and reconstructed with an isotropic voxel spacing of $150 \mu \mathrm{m}$. Images were analyzed using MicroView (Parallax Innovations, London, ON) and CT attenuation was reported in Hounsfield Units (HU). All HU values were measured over a volume of $0.09 \mathrm{~mm}^{3}$. The averaged CT attenuation in the bladder (pre-injection) was subtracted from measured tissue attenuations, to report tissue contrast enhancement throughout this paper.

Contrast agent effect on cell viability. An in vitro cell viability assay was performed using C2C12 mouse myoblast cells, which were incubated for $24 \mathrm{~h}$ with contrast agent at concentrations from 0.063 to $1.0 \mathrm{mg} / \mathrm{ml}$. Following incubation, cell viability was measured using a 3-(4,5-dimethylthiazol-2-yl)-2,5-diphenyltetrazolium bromide (MTT) assay. Additional details are provided in the SI.

In vivo characterization. Animal care and handling. All animal studies were carried out in accordance with the regulations set out by the Canadian Council on Animal Care, as per an Animal Use Protocol 2018-001 approved by the University of Western Ontario's Council on Animal Care. C57BL/6 male mice (25-32 g) were purchased from The Jackson Laboratory (Bar Harbor, ME), and were housed in Type II open polycarbonate cage. The animals were kept in temperature and humidity-controlled rooms on a $12 \mathrm{~h}$ light-dark cycle, with regular chow and water available ad libitum.

The animals were anesthetized prior to contrast-agent injection and during micro-CT scans using $3.5 \%$ isoflurane (Forane, Baxter Corporation, Mississauga, $\mathrm{ON}$ ) in $\mathrm{O}_{2}$ via a nose cone, which was reduced to $1.5 \%$ for maintenance.

Subcutaneous tissue reaction test. The in vivo toxicity of the contrast agent was evaluated by injecting $\mathrm{PPEG}_{2000}-\mathrm{GdNP}$ and $\mathrm{PPEG} \mathrm{F}_{500}-\mathrm{PPEG}_{1000}-\mathrm{GdNP}(0.2 \mathrm{~mL}, n=2$ each formulation) subcutaneously into the dorsal interscapular tissue. The animals were scanned immediately after and two weeks post-injection. The animals were euthanized and dissected for gross tissue observations. Additional details are provided in the SI.

Time-course contrast enhancement and biodistribution. The tail veins of seven mice were catheterized using PE-20 polyethylene tubing. $\mathrm{PPEG}_{2000}$-GdNP was injected over a period of $3 \mathrm{~min}(0.2 \mathrm{~mL}, n=4)$ while $\mathrm{PPEG}_{5000}-\mathrm{PPEG}_{1000}-\mathrm{GdNP}(0.2 \mathrm{~mL}, n=3)$ was injected over a period of $5 \mathrm{~min}$. Micro-CT scans of the animals were obtained at $5,10,15,30$, and 60 min post-injection, as well as 2 and 5 days after. The animals were weighed daily during the experiments, until the subjects were euthanized on day 5. Major organs were processed for gadolinium content measurements by ICP-MS and standard histological analyses ${ }^{43}$.

Statistical analysis. All values are reported as means \pm standard deviations. A two-way repeated-measures analysis of variance (ANOVA) was performed in Prism 8 (GraphPad Software Inc., San Diego, CA, USA) for each formulation to evaluate differences between the contrast enhancement in the vasculature, variations between ICP-MS-measured gadolinium content of excised tissues on day 5 , and to measure the effect of intravenous injections to mouse weight. Results were considered statistically significant at $p<0.05$. 
A
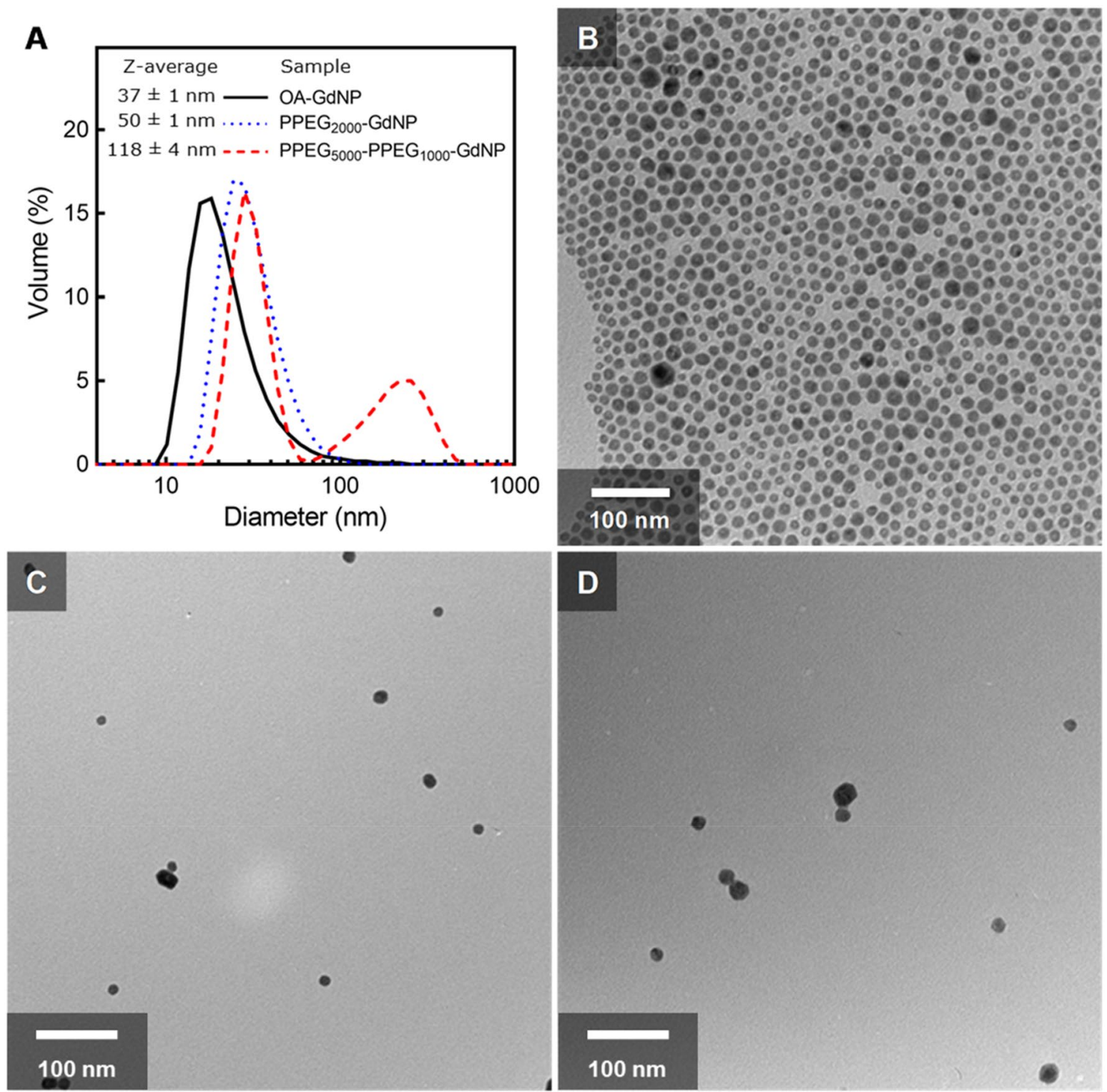

Figure 2. Size distributions and microscopy images of the synthesized GdNPs. (A) DLS volume diameter distributions of OA-GdNP in cyclohexane, and $\mathrm{PEG}_{2000}-\mathrm{GdNP}$ and $\mathrm{PEG}_{5000}-\mathrm{PEG}_{1000}-\mathrm{GdNP}$ in water. Representative TEM images of (B) OA-GdNP, (C) $\mathrm{PEG}_{2000}-\mathrm{GdNP}$ and (D) $\mathrm{PEG}_{5000}-\mathrm{PEG}_{1000}-\mathrm{GdNP}$. The scale bar in (B) applies to all TEM images.

\section{Results}

Physical characterization. $\mathrm{OA}-\mathrm{GdNP}$ and $\mathrm{PPEG}_{2000}-\mathrm{GdNP}$ had monomodal size distributions, with $Z$-average diameters of $37 \pm 1 \mathrm{~nm}$ and $50 \pm 1 \mathrm{~nm}$, respectively, both with polydispersity indices (PDI) of $0.20 \pm 0.01$ (Fig. 2A). $P E_{5000}-P P E G_{1000}-G d N P$ had a bimodal size distribution, with a Z-average diameter of $118 \pm 4 \mathrm{~nm}$ and a PDI of $0.30 \pm 0.01$. The particle diameters measured by DLS were in good agreement with those observed in the TEM images (Fig. 2B-D). After lyophilization and redispersion, $\mathrm{PPEG}_{5000}-\mathrm{PPEG}_{1000}-\mathrm{GdNP}$ had diameters of $202 \pm 8 \mathrm{~nm}$ in saline and $226 \pm 10 \mathrm{~nm}$ in a mouse serum mimic (Fig. 3). Colloidal stability was retained for at least $2 \mathrm{~h}$ with no substantial changes in $\mathrm{Z}$-average diameters. Larger diameters were observed upon redispersion for $P_{P E G_{2000}-G d N P}$ (from $50 \pm 1$ to $354 \pm 99 \mathrm{~nm}$ in saline and $201 \pm 27 \mathrm{~nm}$ in a mouse serum mimic) and the diameters increased over time in saline and mouse serum mimic, as expected.

In the gadolinium release experiment where $\mathrm{PPEG}_{2000}$-GdNP was dialyzed against zinc chloride and calcium chloride in saline at biologically relevant concentrations, some gadolinium release was observed. At the 1 -h time point, $0.4 \%$ of the encapsulated gadolinium was detected in the dialysate. This increased to $3.8 \%$ and $7.1 \%$ after 3 and 5 days, respectively.

ICP-MS analysis of the contrast agents indicated a gadolinium content of $30 \pm 5 \%$ and $22 \pm 3 \%(w / w)$ for $\mathrm{PPEG}_{2000}-\mathrm{GdNP}$ and $\mathrm{PPEG}_{5000}-\mathrm{PPEG}_{1000}-\mathrm{GdNP}$, respectively. A PEG grafting density of about 4 PEG chains per $\mathrm{nm}^{2}$ was calculated for $\mathrm{PPEG}_{2000}$-GdNP (supplementary information), while it was not possible to determine the grafting density for $\mathrm{PPEG}_{5000}-\mathrm{PPEG}_{1000}-\mathrm{GdNP}$ due to the mixture of chain lengths. Hence, to achieve a gadolinium loading of $100 \mathrm{mg} / \mathrm{mL}$ in the contrast agent formulation, $66 \pm 10 \mathrm{mg}$ of dried $\mathrm{PPEG}_{2000}-\mathrm{GdNP}$ or $90 \pm 14 \mathrm{mg}$ of $\mathrm{PPEG}_{5000}-\mathrm{PPEG}_{1000}-\mathrm{GdNP}$ need to be diluted in $200 \mu \mathrm{L}$ saline. The relation between CT contrast 
A

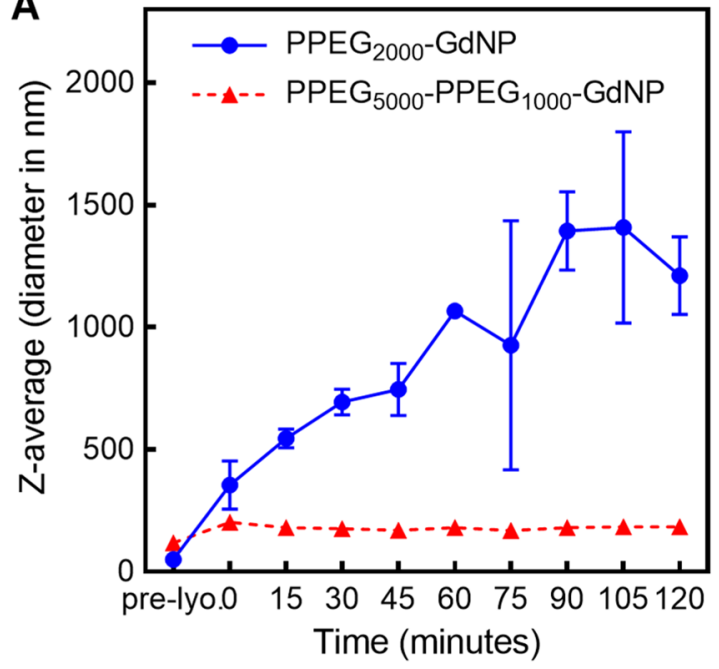

B

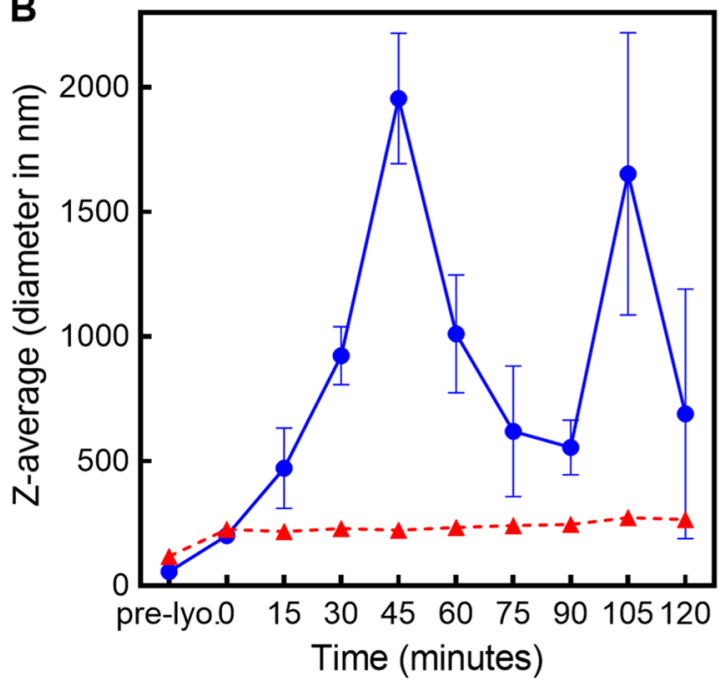

Figure 3. Time-course diameters of resuspended $P P E G_{2000}-G d N P$ and $P P E G_{5000}-P P E G_{1000}-G d N P$ at $37^{\circ} \mathrm{C}$. The contrast agents were redispersed in (A) saline and (B) a mouse serum mimic for characterization.

(HU) and gadolinium concentration in $\mathrm{mg} / \mathrm{mL}$ ([Gd]) was calculated to be $\mathrm{HU}=31[\mathrm{Gd}]-3$ (Supplementary Fig. S1). Hence, a contrast value of $3100 \mathrm{HU}$ is anticipated with $100 \mathrm{mg} / \mathrm{mL}$ of gadolinium. The CT contrast values obtained from $\mathrm{PPEG}_{2000}-\mathrm{GdNP}$ and PPEG ${ }_{5000}-\mathrm{PPEG}_{1000}-\mathrm{GdNP}$ were $3244 \pm 57 \mathrm{HU}$ and $3195 \pm 62 \mathrm{HU}$, respectively, corresponding to $105 \pm 2 \mathrm{mg} / \mathrm{mL}$ and $103 \pm 2 \mathrm{mg} / \mathrm{mL}$ of gadolinium respectively.

In vitro and in vivo characterization. In cell culture experiments with $\mathrm{PPEG}_{2000}-\mathrm{GdNP}$ and $\mathrm{PPEG}_{5000}-\mathrm{PPEG}_{1000}-\mathrm{GdNP}$, greater than $75 \%$ viability of $\mathrm{C} 2 \mathrm{C} 12$ mouse myoblast cells was found for all gadolinium concentrations evaluated, up to $1.0 \mathrm{mg} / \mathrm{mL}$ (Supplementary Fig. S3); the GdNP are not considered toxic according to the American Society for Testing and Materials ${ }^{44}$. In addition, micro-CT images of the dorsal interscapular region following subcutaneous injection (Supplementary Fig. S4) showed contrast enhancement near the injection site was nearly gone after two weeks. Full body gross examinations showed normal tissues.

Representative time-course micro-CT images of mice injected with the GdNPs are shown in Fig. 4. As expected, qualitative evaluation of the post-contrast images shows opacification of the blood pool (vessels, chambers of the heart), which remained high over the $60 \mathrm{~min}$ studied. Increased contrast was observed in the liver as early as 5 -min, as demonstrated by the ability to distinguish the liver from surrounding soft tissues; contrast enhancement increased by the 2 day time point. Liver contrast decreased 5 days after agent injection, accompanied by increased contrast in the spleen. The renal cortex was not distinguishable from surrounding tissues, confirming the evasion of renal clearance and clearance via the RES. Quantitative evaluation of the contrast enhancement in the vasculature and the RES organs is shown in Fig. 5. For PPEG $2000-\mathrm{GdNP}$, an average attenuation of $245 \pm 32 \mathrm{HU}$ was observed 5 min post-injection in the abdominal aorta, while an attenuation of $278 \pm 33 \mathrm{HU}$ was observed for PPEG $5000-\mathrm{PPEG}_{1000}-\mathrm{GdNP}$. Values of $200 \mathrm{HU}$ were observed in the blood pool for at least $30 \mathrm{~min}$ for both formulations. No significant difference between the measured contrast enhancement of the vasculature for up to $60 \mathrm{~min}$, and no effect on attenuation due to PEG chain length were observed (2-way ANOVA, $p=0.15$ and 0.077 , respectively). Following a single-phase decay model, half-lives were calculated to be 194 and $135 \mathrm{~min}$ for PPEG $2000-$ GdNP and PPEG $5000-$ PPEG $_{1000}-$ GdNP, respectively (Supplementary Fig. S2).

The mice were lethargic one day after injection; normal behavior resumed on the second day. The average mass of the injected animals decreased one day after agent injection for both formulations and stopped decreasing after day 3 (Fig. 6). There were no significant differences between daily mass measurements $(p=0.30)$.

Based on post-mortem ICP-MS analyses of the tissues, low gadolinium concentrations were found in the blood, while high gadolinium concentrations were observed in the liver and spleen, as expected (Table 1). The livers and spleens excised from $P_{P E G_{500}}-P_{P E G}{ }_{1000}-G d N P$-injected mice were visibly larger than the organs of mice that were injected with $P P E G_{2000}-G d N P$. While contrast values do not depend on the size or mass of the organ, the total gadolinium contents of the organs were greater for PPEG $5000-P_{P E G}{ }_{1000}-G d N P$ than $P P E G_{2000}-G d N P$ due to organ mass differences $(96 \pm 22 \mu \mathrm{g}$ vs. $58 \pm 14 \mu \mathrm{g}$ for the liver, and $68 \pm 19 \mu \mathrm{g}$ vs. $46 \pm 11 \mu \mathrm{g}$ for the spleen, respectively). No gadolinium was found in the heart, kidneys or bladder, but trace gadolinium was measured in the lungs of mice injected with PPEG $5000-\mathrm{PPEG}_{1000}$-GdNP.

The calibration between CT contrast and gadolinium concentration (Supplementary Fig. S1) confirmed the agreement between HU values (Fig. 5) and the ICP-MS results. The HU values in the liver were calculated to be $298 \pm 66 \mathrm{HU}$ and $345 \pm 108 \mathrm{HU}$ for $\mathrm{PPEG}_{2000}-\mathrm{GdNP}$ and $\mathrm{PPEG}_{5000}-\mathrm{PPEG}_{1000}-\mathrm{GdNP}$, respectively, and were $387 \pm 83 \mathrm{HU}$ and $669 \pm 78 \mathrm{HU}$ in the spleen. The trace amount of gadolinium found in the lungs by ICP-MS were far lower than the noise, and too low to be detected from the CT images. Significant differences in the gadolinium content values between formulations were observed for the liver $(p=0.0006)$ and the spleen $(p<0.0001)$. Of the 

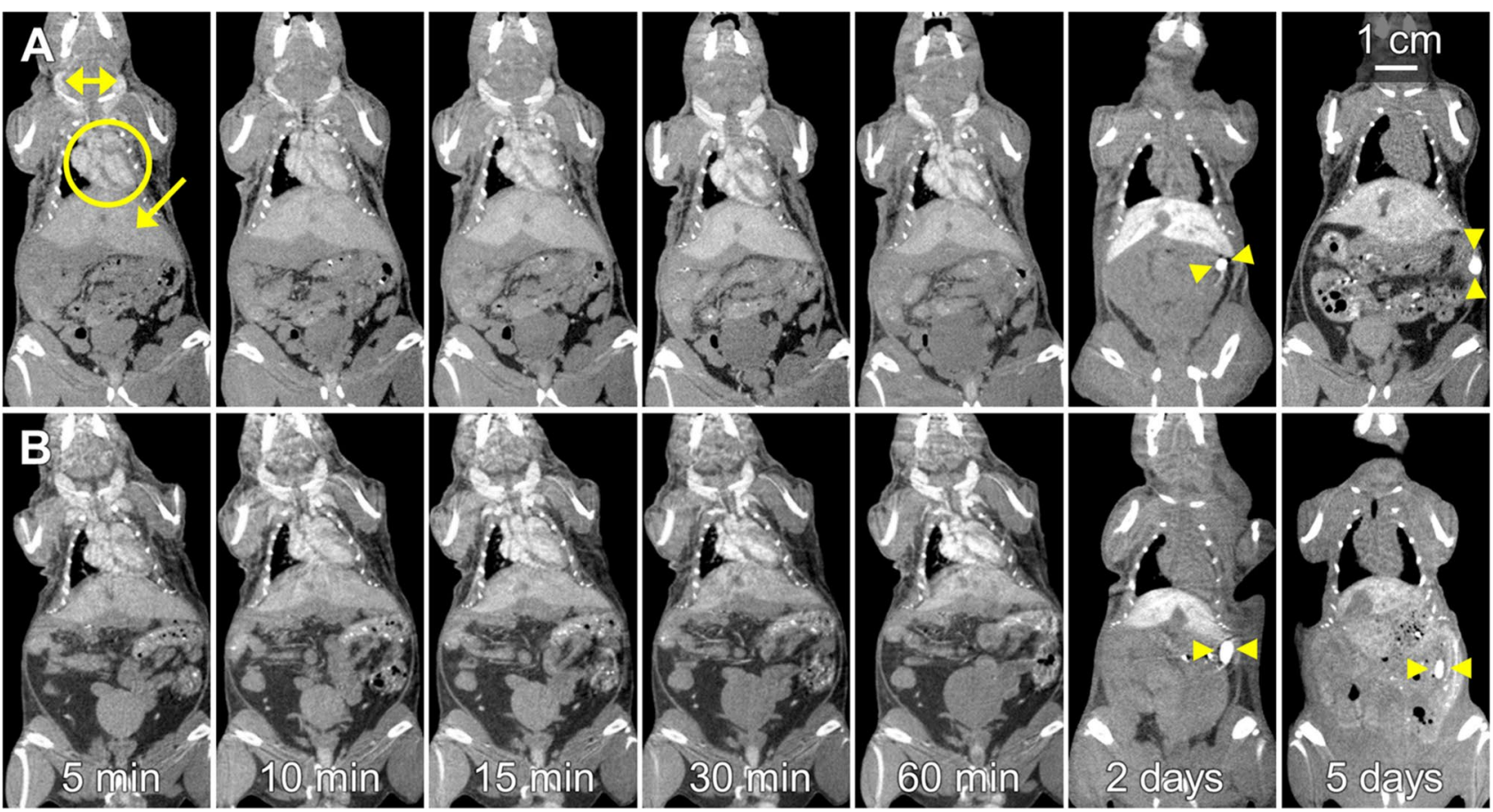

Figure 4. Representative coronal micro-CT images showing the heart, liver, jugular veins, and spleen of mice that received contrast agent formulated with (A) $P_{P E G}{ }_{200}-G d N P$ and (B) $P P E G_{5000}-P_{P E G}{ }_{1000}-G d N P$. All times were reported from the completion of the contrast agent injection. In the 5-min image in (A), the blood in the chambers of the heart (circle), liver (arrow) and the external jugular veins (double arrowheads) are clearly visible. In the 2- and 5-day images, the blood pool in the heart is no longer visible, the liver remains visible, and the spleen (arrowheads) becomes visible. Adjacent anatomical slices were shown for the images acquired within $60 \mathrm{~min}$, while slices located $1 \mathrm{~cm}$ posterior were shown in the 2- and 5-day images, to demonstrate the high contrast in the previously indistinguishable spleen.
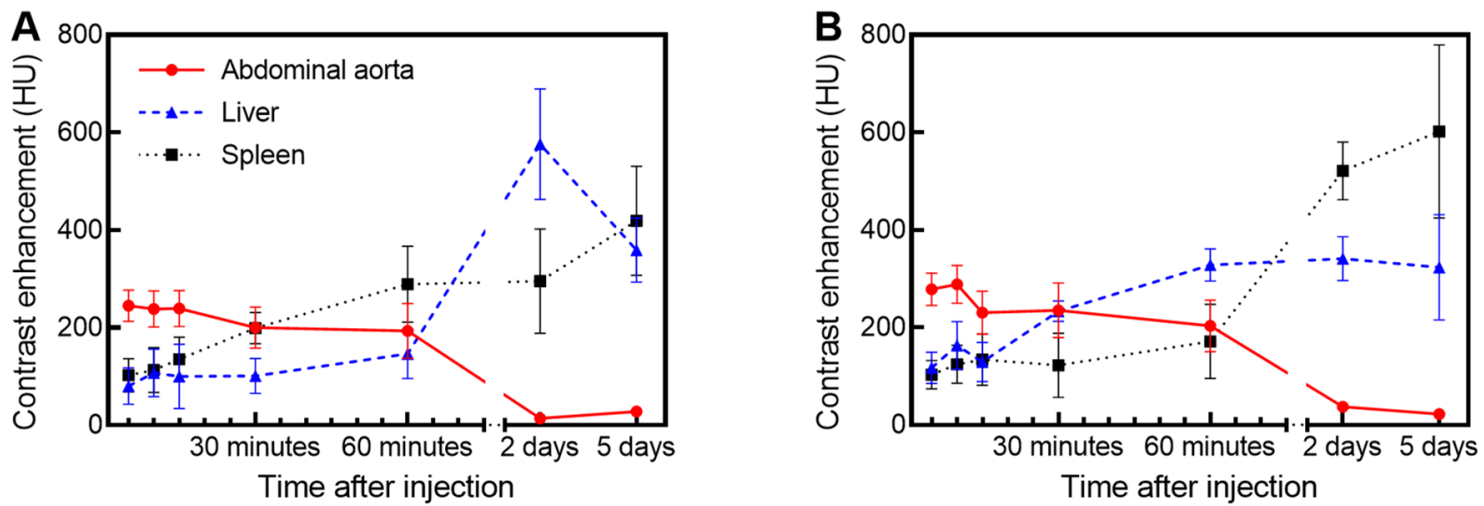

Figure 5. Contrast enhancement in the organs of mice that were injected with (A) $P P E G_{2000}-G d N P$ and (B) $\mathrm{PPEG}_{5000}-\mathrm{PPEG}_{1000}-\mathrm{GdNP}$ displayed similar trends over time, where decreased attenuation in the vasculature was accompanied by increased attenuation in the liver and the spleen.

injected gadolinium, a total of $5.6 \pm 1.0 \%$ and $12.4 \pm 2.1 \%$ remained in the evaluated organs for PPEG ${ }_{2000}-G d N P$ and $\mathrm{PPEG}_{5000}-\mathrm{PPEG}_{1000}-\mathrm{GdNP}$, respectively.

Histological analysis revealed no differences between control and injected mouse liver (Fig. 7), lungs, heart, kidneys and bladder (Supplementary Fig. S5). In spleen tissues, sections from the injected mice demonstrate the presence of basophilic nuclear contents in the cytoplasm and a lower density of nuclei than observed in the control spleen section.

\section{Discussion}

We successfully prepared a long-circulating gadolinium nanoparticle-based vascular micro-CT contrast agent. The nanoparticles can be freeze-dried and redispersed into a contrast agent suspension that is colloidally stable and contains $100 \mathrm{mg} / \mathrm{mL}$ of gadolinium, which is difficult to achieve in an aqueous solution. Whole-body CT 


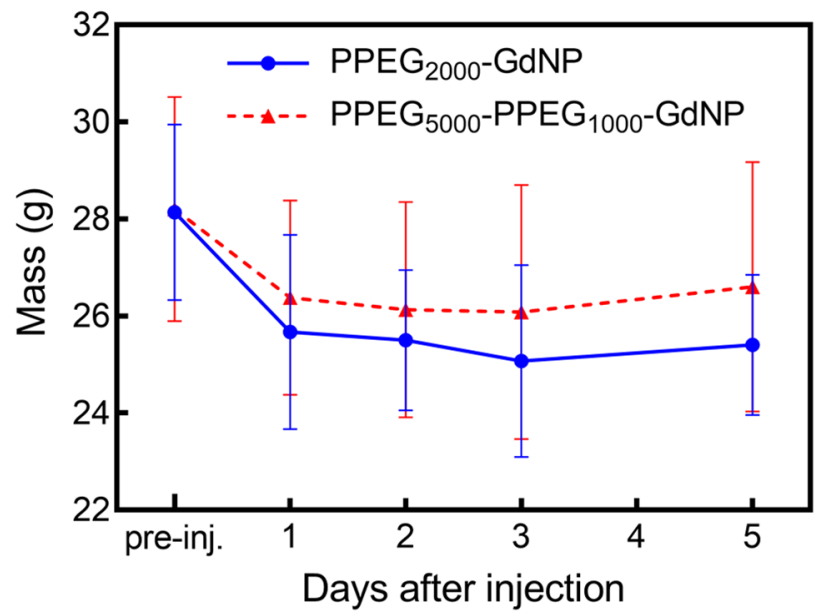

Figure 6. Effect of the contrast-agent injection procedure on subject mass.

\begin{tabular}{|c|c|c|c|c|}
\hline \multirow[b]{2}{*}{ Tissue } & \multicolumn{2}{|c|}{ PPEG $_{2000}-$ GdNP } & \multicolumn{2}{|c|}{ PPEG $_{5000}-$ PPEG $_{1000}-$ GdNP } \\
\hline & ppm & w/w \% of injected gadolinium & ppm & w/w \% of injected gadolinium \\
\hline Blood $^{\mathrm{a}}$ & $1 \pm 31$ & $0.1 \pm 0.2$ & $5 \pm 88$ & $0.1 \pm 0.8$ \\
\hline Lungs & 0 & 0 & $52 \pm 92$ & $0.1 \pm 0.1$ \\
\hline Brain & 0 & 0 & 0 & 0 \\
\hline Liver & $9322 \pm 2060$ & $2.7 \pm 0.6$ & $10,780 \pm 3350$ & $5.2 \pm 1.6$ \\
\hline Spleen & $12,101 \pm 2570$ & $2.8 \pm 0.6$ & $20,833 \pm 2420$ & $7.0 \pm 0.8$ \\
\hline
\end{tabular}

Table 1. Gadolinium content of mouse blood and clearance organs determined by ICP-MS. ${ }^{a}$ Reported w/w\% were rounded up.

images demonstrate sufficient vascular enhancement $(>200 \mathrm{HU})$ over the time period required for scanning, and along with histology and post mortem ICP-MS, confirm RES clearance of both agents. We report the condition of the RES organs days after a high-dose intravenous injection of lanthanide nanoparticles, which has scarcely been reported for other agents. Our results are consistent with an extensive study that demonstrated the performance and in vivo fate of alkaline-earth metal and iodinated nanoparticles when used as vascular contrast agents for micro-CT ${ }^{45}$. Our agent provides comparable vascular contrast and similar clearance pathways as alkaline-earth metal nanoparticles, including their localization in RES organs post-circulation.

Phosphate-terminated PEG was selected to replace the surface oleic acid moieties and render the OA-GdNPs dispersible in water. The phosphate group has higher affinity for $\mathrm{Gd}^{3+}$ compared to the carboxylic acid of oleic acid, and the binding of PPEG to the surface of analogous $\mathrm{NaYF}_{4}$ nanoparticles has been previously demonstrated using ${ }^{31} \mathrm{P}$ NMR spectroscopy ${ }^{41}$. We selected $\mathrm{PPEG}_{2000}$ and $\mathrm{PPEG}_{5000}$ for this study since these are two of the most commonly used PEG chain lengths in long circulating nanoparticles ${ }^{46,47}$. The grafting density of the $\mathrm{PPEG}_{2000}-\mathrm{GdNP}$ was similar to that observed for gold nanoparticles coated with PEG thiols ${ }^{48}$. While the original goal was to compare the performance of $\mathrm{PPEG}_{2000}-\mathrm{GdNP}$ and $\mathrm{PPEG}_{5000^{-}} \mathrm{GdNP}$, our preliminary studies showed that $P_{P E G}{ }_{5000}-G d N P$ could not be redispersed adequately in aqueous solution. It was suspected that exchange of $\mathrm{PPEG}_{5000}$ chains onto the GdNP was more difficult than for $\mathrm{PPEG}_{2000}$, thus leaving some of the hydrophobic OA-coated GdNP surface exposed. In attempts to improve the coverage of the GdNP surface, excess PPEG $_{5000}$ of up to five times the mass of the GdNP was used when preparing PPEG ${ }_{5000}-\mathrm{GdNP}$. However, the nanoparticles could still not be entirely redispersed in water. Backfilling with a shorter PEG chain length was then adopted, which previous studies have demonstrated to reduce interactions between nanoparticles and plasma proteins ${ }^{42}$. To backfill, PPEG $_{1000}$ was selected as this PEG length was not expected to interfere with the performance of $\mathrm{PPEG}_{5000}$ in repelling plasma proteins. The lyophilized $P \mathrm{PEG}_{5000}-\mathrm{PPEG}_{1000}-\mathrm{GdNP}$ redispersed without difficulty in saline and the mouse serum mimic to form particles with average diameters that were no more than twice the pre-lyophilization diameters. $\mathrm{PPEG}_{2000}$-GdNP redispersed at about sevenfold higher diameter than pre-lyophilization. It should be noted that similar $\mathrm{PPEG}_{2000}$ coated $\mathrm{NaYF}_{4}$ particles were found to be stable with respect to aggregation for weeks to months when incubated in water or cell culture media. However, the lyophilization process that we used, which is needed to obtain the high concentrations required for CT, may hinder our ability to completely redisperse the dry materials into individual particles in solution, and may expose hydrophobic sites that lead to gradual aggregation ${ }^{41}$.

The PPEG ${ }_{5000}-\mathrm{PPEG}_{1000}$-GdNP formulation was observed (qualitatively) to have a higher viscosity than $\mathrm{PPEG}_{2000}-\mathrm{GdNP}$ at concentrations of $100 \mathrm{mg} / \mathrm{mL}$ of gadolinium. This resulted in longer injection times for 

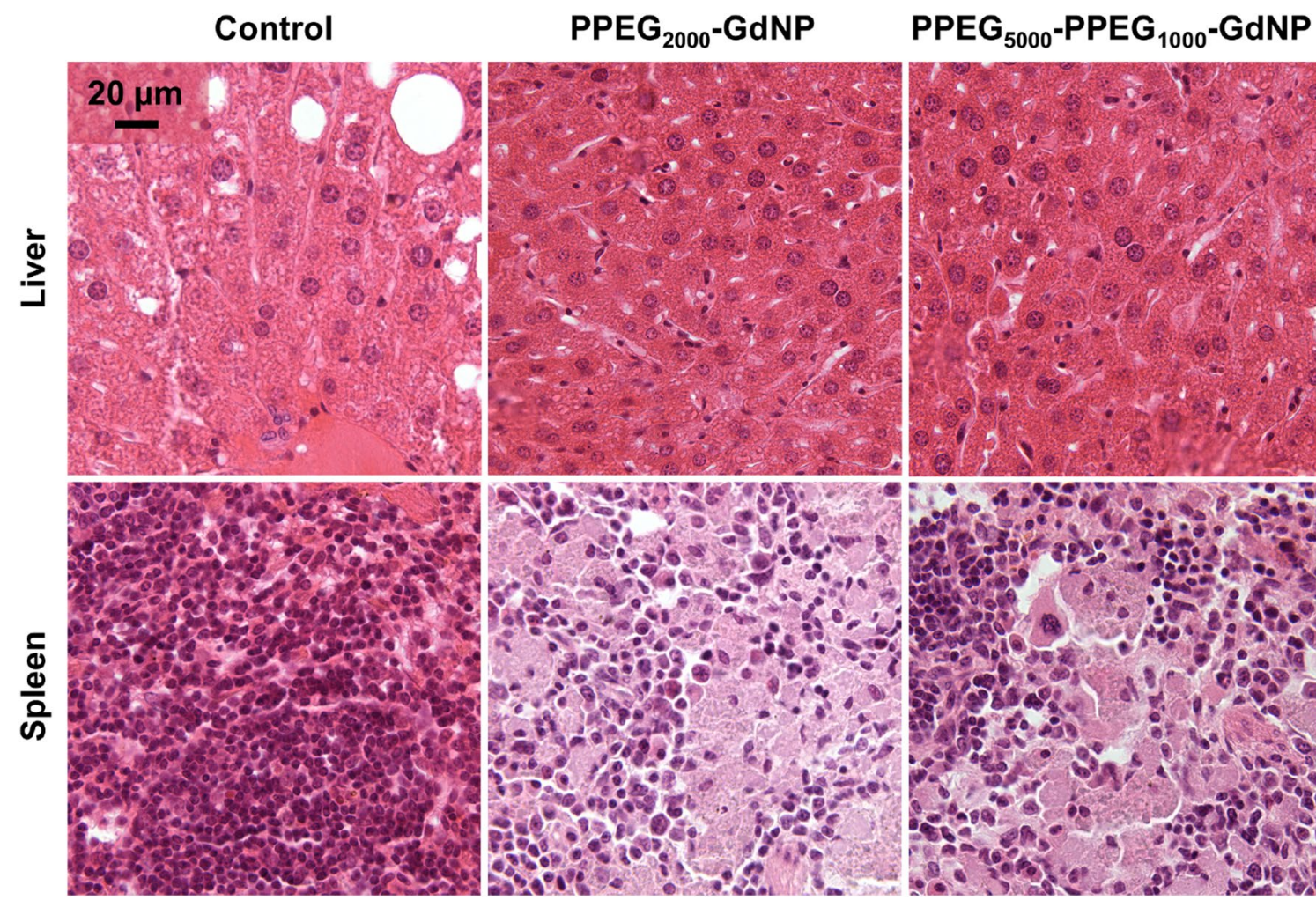

Figure 7. Representative histology images for control and injected mice taken from tissues after 5 days of injection. The scale bar in the liver control section applies to all images. Liver tissues appear normal, while the spleen tissues of the injected mice indicate basophilic nuclear contents in the cytoplasm and lower nuclear densities than the control tissue.

$\mathrm{PPEG}_{5000}-\mathrm{PPEG}_{1000}$-GdNP, requiring 5-min injections versus the 3-min injections when using $\mathrm{PPEG}_{2000}-\mathrm{GdNP}_{\text {. }}$ The PPEG ${ }_{5000}-\mathrm{PPEG}_{1000}$-GdNP had a lower gadolinium content $(22 \pm 3 \% \mathrm{w} / \mathrm{w})$ compared to the $\mathrm{PPEG}_{2000}-\mathrm{GdNP}$ $(30 \pm 5 \%)$. Therefore, more $\mathrm{PPEG}_{5000}-\mathrm{PPEG}_{1000}-\mathrm{GdNP}$ on a mass/volume ratio was required to achieve $100 \mathrm{mg} /$ $\mathrm{mL}$ of gadolinium for the injection. Since the viscosity of a suspension containing solid spherical particles increases when a larger volume of the solution is occupied by the particles/polymer, the viscosity was higher for $\mathrm{PPEG}_{5000}-\mathrm{PPEG}_{1000}-\mathrm{GdNP}^{49}$. The longer $\mathrm{PPEG}_{5000}$ chains on the particle surface may also contribute to higher viscosity compared to $\mathrm{PPEG}_{2000}$.

Similar contrast enhancement of the vasculature (above $200 \mathrm{HU}$ ) was achieved for $30 \mathrm{~min}$ after injection for both formulations, with no significant change in time-course attenuation for up to $60 \mathrm{~min}$. The similarity in in vivo performance of the reported formulations was initially unexpected based on the in vitro studies in saline and the mouse serum mimic because $P_{P E G}{ }_{2000}-G d N P$ showed poorer colloidal stability than $\mathrm{PPEG}_{5000}-\mathrm{PPEG}_{1000}-\mathrm{GdNP}$. However, the in vivo behavior of nanoparticles is largely determined by the protein corona, a complex multilayered structure, with a composition that is determined by the initial nanoparticle surface chemistry as well as factors such as sheer stresses experienced in the blood and extravasation within blood vessels $s^{50,51}$. Protein coronas differ between in vitro and in vivo studies so in vitro tests cannot fully predict the in vivo behavior of nanoparticles in the vasculature. The nanoparticles reported in this paper exhibited the same circulation times and blood pool contrast enhancements as our previous formulation comprising $\mathrm{NaErF}_{4}$ nanoparticles encapsulated in assemblies formed from PEG-poly(lactic acid) block copolymers ${ }^{40}$, which also had PEG surfaces. This current study and our previous paper are further testaments to the capabilities of PEG in temporarily promoting stealth properties against the RES and in providing its cargo with long circulation times in the vasculature, even at the high doses required for micro-CT.

No CT scans were performed after the first $60 \mathrm{~min}$ on day one or the day after injections to avoid complicating mouse health due to anesthesia ${ }^{52}$. The injected mice were clearly lethargic after being under anesthesia for 60 min, and this effect was still observed after one day. Two days following initial injection, the mice were placed under anesthesia for $5 \mathrm{~min}$, only to permit micro-CT imaging; the mice were more active before and after imaging, and only needed a few minutes to recover from anesthesia. The daily mass measurements exhibit a similar trend that coincides with mouse activity, food and water intake. While a limitation of this study is that no sham study was performed to differentiate between the effect of the agent and the anesthesia, isoflurane was found to increase mouse latency in previous studies and coincided with decreased animal weight for up to 2 days ${ }^{53}$.

Our results-micro-CT time course and post-mortem ICP-MS of relevant organs-clearly demonstrate that the agent's clearance pathway is through the RES. This was expected due to its physical properties and is similar to the clearance of other agents incorporating metal nanoparticles ${ }^{32,36}$. Some gadolinium was detected by ICP-MS in 
trace amounts in the blood, and in the lungs of $\mathrm{PPEG}_{5000}-\mathrm{PPEG}_{1000}-\mathrm{GdNP}$-injected mice; the trace values found in the lungs can be attributed to reversible transient aggregation in capillary beds that other intravenously injected nanoparticles also demonstrated ${ }^{54,55}$. Because of concerns with free gadolinium being observed in humans post small-molecule contrast injection ${ }^{56,57}$, we evaluated the gadolinium concentration in the brain and confirmed that our GdNP did not cross the blood brain barrier.

The ICP-MS results reported significantly higher gadolinium accumulation in the liver and in the spleen from $P_{P E G}{ }_{5000}-P_{P E G}{ }_{1000}-G d N P$ than $P_{P E G} P_{200}-G d N P$. This can be attributed to a difference in PEG properties once grafted onto nanoparticle surfaces, specifically the morphology and grafting density, which was previously reported with gold nanoparticles ${ }^{58,59}$. Considering greater RES organ accumulation, higher viscosity, and heavier excised livers and spleens from $\mathrm{PPEG}_{5000}-\mathrm{PPEG}_{1000}-\mathrm{GdNP}$ injections than from $\mathrm{PPEG}_{2000}-\mathrm{GdNP}$, and no significant difference in time-course contrast enhancements of the vasculature, a greater advantage can be gained from using $\mathrm{PPEG}_{2000}-\mathrm{GdNP}$ instead of $\mathrm{PPEG}_{5000}-\mathrm{PPEG}_{1000}-\mathrm{GdNP}$ for in vivo micro-CT. This was an unexpected result, because $P P E G_{5000}-P_{P E G} 1000-G d N P$ particle suspensions were more stable in vitro. We suspect that the size transience of $\mathrm{PPEG}_{2000}-\mathrm{GdNP}$ - demonstrated within minutes of incubation with saline and a mouse serum mimic-promoted mobility and reduced accumulation in RES organs. This has been previously demonstrated by other researchers that studied nanogels of varying rigidity, reporting greater particle mobility and lower accumulation in clearance organs with less rigid nanogels compared to more rigid nanogels ${ }^{60,61}$.

Optical histology results showed that all tissues examined, except for the spleen, were normal. The gadolinium release experiment demonstrated the possibility of released ions causing toxicity in the spleen, although we did perform the experiments using concentrated salts in the dialysate. Nonetheless, the particles are much more stable with respect to the release of free gadolinium ions than small molecule gadolinium agents.

In this study, mice were euthanized after the 5-day time point because after that time point it is difficult to gauge if well-being is a result of the contrast agent or other factors. The contrast agent formulation that we report in this paper presents an improvement from our previously reported nanoparticle assembly formulation, where the majority of mice died after 2 days. While further studies are required to enable the contrast agent's utility in long-term studies, our results show that switching from diblock polymer coated nanoparticle assemblies to grafting PEG directly on the surface-preferably with $\mathrm{PEG}_{2000}$-facilitated the exit of nanoparticles from the liver, which will ultimately be important to ensure that the particles are cleared from the body before the eventual breakdown of the nanoparticles and the potential release of $\mathrm{Gd}^{3+}$ ions.

\section{Conclusions}

We synthesized long-circulating contrast agents composed of PEG-modified GdNP that can be suspended at $100 \mathrm{mg} / \mathrm{mL}$ of gadolinium and can be used to visualize the vasculature of live mice using micro-CT for up to $60 \mathrm{~min}$. The alternative approach of modifying the surface of the nanoparticles with PEG in lieu of polymercoated nanoparticle assemblies presents a step in the right direction towards making micro-CT contrast agents available for long-term longitudinal vascular research.

\section{Data availability}

Data sets are available upon request from the corresponding author.

Received: 30 March 2021; Accepted: 9 July 2021

Published online: 16 August 2021

\section{References}

1. Dobnikar, L. et al. Disease-relevant transcriptional signatures identified in individual smooth muscle cells from healthy mouse vessels. Nat. Commun. 9, 4567. https://doi.org/10.1038/s41467-018-06891-x (2018).

2. Gordon-Keylock, S., Sobiesiak, M., Rybtsov, S., Moore, K. \& Medvinsky, A. Mouse extraembryonic arterial vessels harbor precursors capable of maturing into definitive HSCs. Blood 122, 2338-2345. https://doi.org/10.1182/blood-2012-12-470971 (2013).

3. Trogan, E. et al. Serial studies of mouse atherosclerosis by in vivo magnetic resonance imaging detect lesion regression after correction of dyslipidemia. Arterioscler. Thromb. Vasc. Biol. 24, 1714-1719. https://doi.org/10.1161/01.ATV.0000139313.69015.1c (2004).

4. Vafaie, F. et al. Collagenase-resistant collagen promotes mouse aging and vascular cell senescence. Aging Cell 13, 121-130. https:// doi.org/10.1111/acel.12155 (2014).

5. Ashton, J. R. et al. Anatomical and functional imaging of myocardial infarction in mice using micro-CT and eXIA 160 contrast agent. Contrast Media Mol. Imaging 9, 161-168. https://doi.org/10.1002/cmmi.1557 (2014).

6. Foster, W. K. \& Ford, N. L. Investigating the effect of longitudinal micro-CT imaging on tumour growth in mice. Phys. Med. Biol. 56, 315-326. https://doi.org/10.1088/0031-9155/56/2/002 (2011).

7. Ghanavati, S., Yu, L. X., Lerch, J. P. \& Sled, J. G. A perfusion procedure for imaging of the mouse cerebral vasculature by X-ray micro-CT. J. Neurosci. Methods 221, 70-77. https://doi.org/10.1016/j.jneumeth.2013.09.002 (2014).

8. Schambach, S. J., Bag, S., Groden, C., Schilling, L. \& Brockmann, M. A. Vascular imaging in small rodents using micro-CT. Methods 50, 26-35. https://doi.org/10.1016/j.ymeth.2009.09.003 (2010).

9. Wong, M. D., Dorr, A. E., Walls, J. R., Lerch, J. P. \& Henkelman, R. M. A novel 3D mouse embryo atlas based on micro-CT. Development 139, 3248-3256. https://doi.org/10.1242/dev.082016 (2012).

10. Ehling, J., Lammers, T. \& Kiessling, F. Non-invasive imaging for studying anti-angiogenic therapy effects. Thromb. Haemost. 109, 375-390. https://doi.org/10.1160/TH12-10-0721 (2013).

11. Munce, N. R. et al. Intravascular and extravascular microvessel formation in chronic total occlusions a micro-CT imaging study. JACC Cardiovasc. Imaging 3, 797-805. https://doi.org/10.1016/j.jcmg.2010.03.013 (2010).

12. Udagawa, A. et al. Micro-CT observation of angiogenesis in bone regeneration. Clin. Oral Implants Res. 24, 787-792. https://doi. org/10.1111/j.1600-0501.2012.02458.x (2013).

13. Brede, C. \& Labhasetwar, V. Applications of nanoparticles in the detection and treatment of kidney diseases. Adv. Chronic Kidney Dis. 20, 454-465. https://doi.org/10.1053/j.ackd.2013.07.006 (2013). 
14. Choi, C. H., Zuckerman, J. E., Webster, P. \& Davis, M. E. Targeting kidney mesangium by nanoparticles of defined size. Proc. Natl. Acad. Sci. U. S. A. 108, 6656-6661. https://doi.org/10.1073/pnas.1103573108 (2011).

15. Gomez-Vallejo, V. et al. PEG-copolymer-coated iron oxide nanoparticles that avoid the reticuloendothelial system and act as kidney MRI contrast agents. Nanoscale 10, 14153-14164. https://doi.org/10.1039/c8nr03084g (2018).

16. Petros, R. A. \& DeSimone, J. M. Strategies in the design of nanoparticles for therapeutic applications. Nat. Rev. Drug Discov. 9, 615-627. https://doi.org/10.1038/nrd2591 (2010).

17. Watermann, A. \& Brieger, J. Mesoporous silica nanoparticles as drug delivery vehicles in cancer. Nanomaterials (Basel) https:// doi.org/10.3390/nano7070189 (2017).

18. Blanco, E., Shen, H. \& Ferrari, M. Principles of nanoparticle design for overcoming biological barriers to drug delivery. Nat. Biotechnol. 33, 941-951. https://doi.org/10.1038/nbt.3330 (2015).

19. Detombe, S. A. et al. Longitudinal follow-up of cardiac structure and functional changes in an infarct mouse model using retrospectively gated micro-computed tomography. Invest. Radiol. 43, 520-529. https://doi.org/10.1097/RLI.0b013e3181727519 (2008).

20. Willekens, I. et al. Time-course of contrast enhancement in spleen and liver with Exia 160, Fenestra LC, and VC. Mol. Imaging Biol. 11, 128-135. https://doi.org/10.1007/s11307-008-0186-8 (2009).

21. Boll, H. et al. Comparison of Fenestra LC, ExiTron nano 6000, and ExiTron nano 12000 for micro-CT imaging of liver and spleen in mice. Acad. Radiol. 20, 1137-1143. https://doi.org/10.1016/j.acra.2013.06.002 (2013).

22. Rothe, J. H. et al. Time course of contrast enhancement by micro-CT with dedicated contrast agents in normal mice and mice with hepatocellular carcinoma: Comparison of one iodinated and two nanoparticle-based agents. Acad. Radiol. 22, 169-178. https:// doi.org/10.1016/j.acra.2014.07.022 (2015).

23. Butcher, N. J., Mortimer, G. M. \& Minchin, R. F. Drug delivery: Unravelling the stealth effect. Nat. Nanotechnol. 11, 310-311. https://doi.org/10.1038/nnano.2016.6 (2016).

24. Yang, Q. et al. Evading immune cell uptake and clearance requires PEG grafting at densities substantially exceeding the minimum for brush conformation. Mol. Pharmacol. 11, 1250-1258. https://doi.org/10.1021/mp400703d (2014).

25. Xiao, R. Z. et al. Recent advances in PEG-PLA block copolymer nanoparticles. Int. J. Nanomed. 5, 1057-1065. https://doi.org/10. 2147/IJN.S14912 (2010).

26. Zhao, Q. et al. PEGylated mesoporous silica as a redox-responsive drug delivery system for loading thiol-containing drugs. Int. J. Pharm. 477, 613-622. https://doi.org/10.1016/j.ijpharm.2014.10.056 (2014).

27. Detombe, S. A., Dunmore-Buyze, J. \& Drangova, M. Evaluation of eXIA 160 cardiac-related enhancement in C57BL/6 and BALB/c mice using micro-CT. Contrast Media Mol. Imaging 7, 240-246. https://doi.org/10.1002/cmmi.488 (2012).

28. Vandeghinste, B. et al. Replacing vascular corrosion casting by in vivo micro-CT imaging for building 3D cardiovascular models in mice. Mol. Imaging Biol. 13, 78-86. https://doi.org/10.1007/s11307-010-0335-8 (2011).

29. Clark, D. P., Ghaghada, K., Moding, E. J., Kirsch, D. G. \& Badea, C. T. In vivo characterization of tumor vasculature using iodine and gold nanoparticles and dual energy micro-CT. Phys. Med. Biol. 58, 1683-1704. https://doi.org/10.1088/0031-9155/58/6/1683 (2013).

30. Domey, J., Teichgraber, U. \& Hilger, I. Gold nanoparticles allow detection of early-stage edema in mice via computed tomography imaging. Int. J. Nanomed. 10, 3803-3814. https://doi.org/10.2147/IJN.S77383 (2015).

31. Hainfeld, J. F. et al. Micro-CT enables microlocalisation and quantification of Her2-targeted gold nanoparticles within tumour regions. Br. J. Radiol. 84, 526-533. https://doi.org/10.1259/bjr/42612922 (2011).

32. Chakravarty, S. et al. Tantalum oxide nanoparticles as versatile contrast agents for X-ray computed tomography. Nanoscale 12, 7720-7734. https://doi.org/10.1039/D0NR01234C (2020).

33. Cardinal, H. N., Holdsworth, D. W., Drangova, M., Hobbs, B. B. \& Fenster, A. Experimental and theoretical x-ray imaging performance comparison of iodine and lanthanide contrast agents. Med. Phys. 20, 15-31. https://doi.org/10.1118/1.597134 (1993).

34. Niranjana, K. M. \& Badiger, N. M. K shell parameters of some lanthanide elements using bremsstrahlung. Radiat. Phys. Chem. 107, 59-64. https://doi.org/10.1016/j.radphyschem.2014.09.013 (2015).

35. Tse, J. J., Dunmore-Buyze, J., Drangova, M. \& Holdsworth, D. W. Dual-energy computed tomography using a gantry-based preclinical cone-beam microcomputed tomography scanner. J. Med. Imaging (Bellingham) 5, 033503. https://doi.org/10.1117/1.JMI.5. 3.033503 (2018).

36. Liu, Y. et al. A high-performance ytterbium-based nanoparticulate contrast agent for in vivo X-ray computed tomography imaging. Angew. Chem. Int. Ed. Engl. 51, 1437-1442. https://doi.org/10.1002/anie.201106686 (2012).

37. Zhou, J., Zhu, X., Chen, M., Sun, Y. \& Li, F. Water-stable NaLuF4-based upconversion nanophosphors with long-term validity for multimodal lymphatic imaging. Biomaterials 33, 6201-6210. https://doi.org/10.1016/j.biomaterials.2012.05.036 (2012).

38. Badea, C. T. et al. Functional imaging of tumor vasculature using iodine and gadolinium-based nanoparticle contrast agents: A comparison of spectral micro-CT using energy integrating and photon counting detectors. Phys. Med. Biol. 64, 065007. https:// doi.org/10.1088/1361-6560/ab03e2 (2019).

39. Bridot, J. L. et al. Hybrid gadolinium oxide nanoparticles: Multimodal contrast agents for in vivo imaging. J. Am. Chem. Soc. 129, 5076-5084. https://doi.org/10.1021/ja068356j (2007).

40. Cruje, C. et al. Polymer assembly encapsulation of lanthanide nanoparticles as contrast agents for in vivo micro-CT. Biomacromol https://doi.org/10.1021/acs.biomac.7b01685 (2018).

41. Boyer, J. C., Manseau, M. P., Murray, J. I. \& van Veggel, F. C. Surface modification of upconverting NaYF4 nanoparticles with PEG-phosphate ligands for NIR (800 nm) biolabeling within the biological window. Langmuir 26, 1157-1164. https://doi.org/10. 1021/la902260j (2010).

42. Dai, Q., Walkey, C. \& Chan, W. C. Polyethylene glycol backfilling mitigates the negative impact of the protein corona on nanoparticle cell targeting. Angew. Chem. Int. Ed. Engl. 53, 5093-5096. https://doi.org/10.1002/anie.201309464 (2014).

43. Bancroft, J. D. \& Gamble, M. (eds) Theory and Practice of Histological Techniques 6th edn. (Churchill Livingstone Elsevier, 2008).

44. Lewinski, N., Colvin, V. \& Drezek, R. Cytotoxicity of nanoparticles. Small 4, 26-49. https://doi.org/10.1002/smll.200700595 (2008).

45. Mannheim, J. G. et al. Comparison of small animal CT contrast agents. Contrast Media Mol. Imaging 11, 272-284. https://doi.org/ $10.1002 / \mathrm{cmmi} .1689$ (2016)

46. Gill, K. K., Kaddoumi, A. \& Nazzal, S. PEG-lipid micelles as drug carriers: Physiochemical attributes, formulation principles and biological implication. J. Drug Target. 23, 222-231. https://doi.org/10.3109/1061186X.2014.997735 (2015).

47. Jokerst, J. V., Lobovkina, T., Zare, R. N. \& Gambhir, S. S. Nanoparticle PEGylation for imaging and therapy. Nanomedicine (London) 6, 715-728. https://doi.org/10.2217/nnm.11.19 (2011).

48. Benoit, D. N. et al. Measuring the grafting density of nanoparticles in solution by analytical ultracentrifugation and total organic carbon analysis. Anal. Chem. 84, 9238-9245. https://doi.org/10.1021/ac301980a (2012).

49. Xu, F. et al. The calculation scheme for prediction of viscosity for polymeric nano-suspensions. AIP Conf. Proc. 1839, 020005. https://doi.org/10.1063/1.4982370 (2017).

50. Zhang, Y., Wu, J. L. Y., Lazarovits, J. \& Chan, W. C. W. An analysis of the binding function and structural organization of the protein corona. J. Am. Chem. Soc. 142, 8827-8836. https://doi.org/10.1021/jacs.0c01853 (2020).

51. Ke, P. C., Lin, S., Parak, W. J., Davis, T. P. \& Caruso, F. A decade of the protein corona. ACS Nano 11, 11773-11776. https://doi. org/10.1021/acsnano.7b08008 (2017).

52. Hankenson, F. C., Braden-Weiss, G. C. \& Blendy, J. A. Behavioral and activity assessment of laboratory mice (Mus musculus) after tail biopsy under isoflurane anesthesia. J. Am. Assoc. Lab Anim Sci 50, 686-694 (2011). 
53. Hohlbaum, K. et al. Severity classification of repeated isoflurane anesthesia in C57BL/6JRj mice-Assessing the degree of distress. PLoS ONE 12, e0179588. https://doi.org/10.1371/journal.pone.0179588 (2017).

54. Kutscher, H. L. et al. Threshold size for optimal passive pulmonary targeting and retention of rigid microparticles in rats. J. Control Release 143, 31-37. https://doi.org/10.1016/j.jconrel.2009.12.019 (2010).

55. Lee, S. Y. et al. Transient aggregation of chitosan-modified poly(d, l-lactic-co-glycolic) acid nanoparticles in the blood stream and improved lung targeting efficiency. J. Colloid Interface Sci. 480, 102-108. https://doi.org/10.1016/j.jcis.2016.07.006 (2016).

56. McDonald, R. J. et al. Intracranial gadolinium deposition after contrast-enhanced MR imaging. Radiology 275, 772-782. https:// doi.org/10.1148/radiol.15150025 (2015).

57. Gulani, V. et al. Gadolinium deposition in the brain: Summary of evidence and recommendations. Lancet Neurol. 16, 564-570. https://doi.org/10.1016/S1474-4422(17)30158-8 (2017).

58. Hoshyar, N., Gray, S., Han, H. \& Bao, G. The effect of nanoparticle size on in vivo pharmacokinetics and cellular interaction. Nanomedicine (London) 11, 673-692. https://doi.org/10.2217/nnm.16.5 (2016).

59. Lipka, J. et al. Biodistribution of PEG-modified gold nanoparticles following intratracheal instillation and intravenous injection. Biomaterials 31, 6574-6581. https://doi.org/10.1016/j.biomaterials.2010.05.009 (2010).

60. Merkel, T. J. et al. The effect of particle size on the biodistribution of low-modulus hydrogel PRINT particles. J. Control Release 162, 37-44. https://doi.org/10.1016/j.jconrel.2012.06.009 (2012).

61. Zhang, L. et al. Softer zwitterionic nanogels for longer circulation and lower splenic accumulation. ACS Nano 6, 6681-6686. https:// doi.org/10.1021/nn301159a (2012).

\section{Acknowledgements}

This work was funded by a grant from the Heart and Stroke Foundation of Canada (G-14-0005959). Funding support also came from the Canadian Institutes of Health Research (FDN-143326). The authors would like to thank the Biotron imaging center at Western University for allowing us to utilize their instruments. The authors also thank J. Hadway for helpful discussion and assistance with mouse injections.

\section{Author contributions}

C.C., D.W.H., E.R.G. and M.D. conceived the experiments. C.C., P.J.D. and E.G. conducted the experiments; C.C. wrote the manuscript and prepared the figures. All authors participated in discussions regarding the methodology and interpretation of the results and reviewed and revised the manuscript for critical intellectual input.

\section{Competing interests}

The authors declare no competing interests.

\section{Additional information}

Supplementary Information The online version contains supplementary material available at https://doi.org/ 10.1038/s41598-021-95716-X.

Correspondence and requests for materials should be addressed to M.D.

Reprints and permissions information is available at www.nature.com/reprints.

Publisher's note Springer Nature remains neutral with regard to jurisdictional claims in published maps and institutional affiliations.

(c) (i) Open Access This article is licensed under a Creative Commons Attribution 4.0 International License, which permits use, sharing, adaptation, distribution and reproduction in any medium or format, as long as you give appropriate credit to the original author(s) and the source, provide a link to the Creative Commons licence, and indicate if changes were made. The images or other third party material in this article are included in the article's Creative Commons licence, unless indicated otherwise in a credit line to the material. If material is not included in the article's Creative Commons licence and your intended use is not permitted by statutory regulation or exceeds the permitted use, you will need to obtain permission directly from the copyright holder. To view a copy of this licence, visit http://creativecommons.org/licenses/by/4.0/.

(c) The Author(s) 2021 